\title{
The Challenges of Microelectronics for the Future Digital Society: The Roles of Thin Film Technologies and of the Higher Education
}

\author{
O. Bonnaud $1,2,3$ \\ ${ }^{1}$ IETR, University of Rennes 1, Rennes, France \\ ${ }^{2}$ GIP-CNFM, Grenoble, France \\ ${ }^{3}$ Dept. Electrical Engineering, South-East-University, Nanjing, China \\ Email: Olivier.bonnaud@univ-rennes1.fr
}

How to cite this paper: Bonnaud, O. (2019) The Challenges of Microelectronics for the Future Digital Society: The Roles of Thin Film Technologies and of the Higher Education. Journal of Materials Science and Chemical Engineering, 7, 47-56. https://doi.org/10.4236/msce.2019.712006

Received: October 15, 2019 Accepted: December 17, 2019 Published: December 20, 2019

\begin{abstract}
The new digital society is based on Internet of Things (IoT) and related connected objects are becoming more prevalent around the world. This evolution implies innovation in many areas of technology, the heart of which is microelectronics. Connected objects involve many technological components and functions that are directly dependent of the microelectronics capabilities. If the perspectives are exciting, several challenges are appearing. The first is related to the energy consumption of all these objects, which will become enormous by 2030, and unrealistic by 2040 . The second is human resources concern. The future engineers and $\mathrm{PhD}$ will have many obstacles to overcome. A way to face these challenges is to involve more and more new thin film technologies that must be combined with VLSI ones, and to better train students to this domain with enough know-how and with a large spectrum of knowledge suitable for multidisciplinary applications. The French national network for Higher Education in microelectronics has adopted this strategy. After presentation of the challenges, this paper deals with the innovative activities of the French network focused on thin film technologies, in order to face the challenges in a short future.
\end{abstract}

\section{Keywords}

Microelectronics, Thin Film Technologies, Digital Society Challenges, Higher Education, Thin Film Microelectronics Devices and Systems

\section{Introduction}

The evolution towards a digital society and the Internet of Things [1] implies the 
development of a very large number of products [2] or connected objects [3]. These include multiple functions that are integrated and the main ones are sensors, actuators, analog-to-digital or digital-to-analog conversion circuits, signal processing, energy harvesting, integrated transmission and reception modules associated with transmission protocols, as well as all the usual displays, alarm and control elements [4]. These objects can have many applications, in fields as varied as the environment, health, transport, agriculture, energy, communications and security [5]. These objects will also be present in industry 4.0, corresponding to a new industrial revolution in both production and industrial tool management [6]. Usual chips may contain today several billion of elementary devices. The present systems may contain many integrated circuits and thus several hundred billion of elementary devices. Even if the consumption of electrical energy decreases at the level of elementary devices, the total consumption of the systems does not do the same. The first challenge that appears is related to the energy consumption of all these objects, which will become enormous by 2030, and unrealistic by 2040 [7]. A way to minimize this growth consists to involve thin-film technologies, particularly transistor-based technologies, and optoelectronics devices which must meet very different mission profiles depending on performance and field of application [5]. Large-area electronics and associated components are becoming increasingly important in integrated systems and especially in system-in-package (SiP), which corresponds to the stacking of circuits possibly on the same substrate but in any case in the same package [8]. It is this evolution that makes it possible to increase integration beyond Moore's law [9], which was essentially based on reducing the dimensions of elementary components during several decades. The introduction of the third dimension [10] opens a new way to significantly increase integration [8]. Therefore, it is necessary to train young graduates in these thin-film and large-area technologies and in particular thin-film transistors, both on knowledge in general and on know-how.

After a presentation of the challenges related to technology and energy consumption, attention is given to the role of thin-film technologies in this field and to the importance of higher education and practice in acquiring know-how and innovative behavior in new architectures of connected objects.

\section{Connected Objects and Thin Film Technologies}

\subsection{Digital Society and Microelectronics}

The development of connected objects contributes to the Internet of Things [11] which appears to be the spearhead of new societies around the world and more especially for the fourth industrial revolution, Industry 4.0. [12]. The connected objects are mainly based on electronic functions that are manufactured with microelectronic devices. They therefore require skills in all areas of microelectronics: integrated silicon electronics, analog and digital circuits, power components and circuits, high frequency and telecommunication components and circuits, 
energy harvesting circuits and components such as photovoltaic cells [13]. Connected objects also require sensors and actuators. These are based on physical conversion devices such as MEMS, MOEMS, BIOMEMS, allowing to broaden the range of applications [4]. Figure 1 shows the schematic architecture of a typical connected object that consists of a first part on site and a second part remotely [13]. Depending on the distance between the two parts and the performance needs of a data center, the transmission power and communication protocols can be very different.

These circuits and systems are developed in different technologies; integrated silicon technologies, but also and increasingly technologies involving new materials such as binary, ternary and quaternary compound semiconductors, organic molecules, materials with two-dimensional structures such as graphene or phosphorene [14]. Many of these technologies are based on thin film deposition. Their role can contribute to the simplicity of the manufacturing process, reduce the number of manufacturing steps and, for more specific functions, significantly reduce power consumption.

\subsection{Role of Thin Film Technologies in Connected Objects}

The first objective is to decrease the fabrication cost thanks to a simplified process, but a second objective, less visible but of a great importance is to decrease the energy consumption of the final object. The best example is the flat panel displays, based on Organic Light Emitting Diodes (OLED) that decrease significantly the consumption of all the panel displays. In mobile objects (cellphone, iPad, etc.) the adoption of these OLED allowed a significant improvement of the energy autonomy of the concerned objects.

Thus, large-area electronics and associated components are becoming increasingly important in integrated systems and especially in system-in-package ( $\mathrm{SiP}$ ), which corresponds to the stacking of circuits possibly on the same substrate but in any case in the same package. It is possible to integrate energy harvesting elements, super capacitances for energy storage, antennas for transceivers, but also many kinds of sensors. In many cases, it is also possible to create directly on integrated circuits thin film devices, called above-ICs devices that introduce new functions like thin film memories [15].

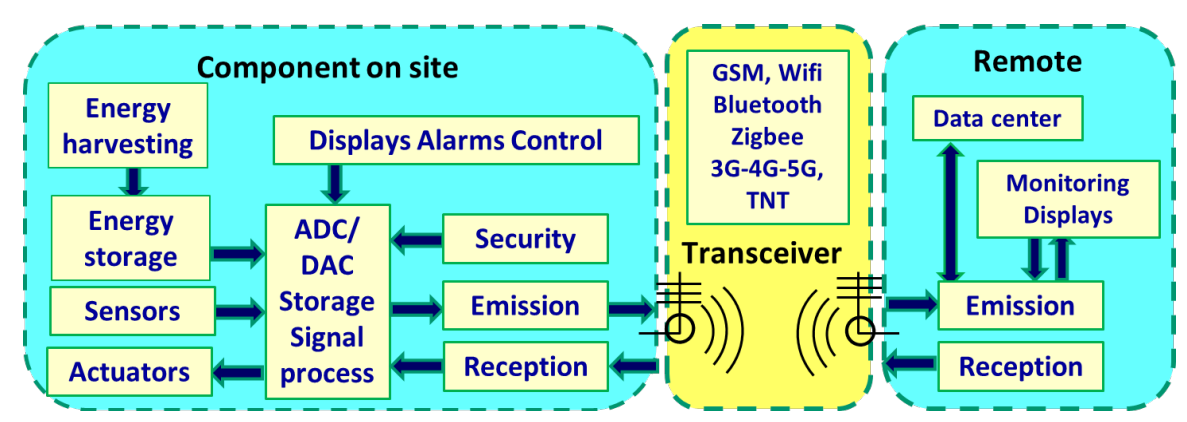

Figure 1. Simplified architecture of a connected object [4]. It includes multiple electronic functions that are fulfilled by thin-film devices. 
Above-ICs approach can be also involved in physical, chemical, and biological sensors with the goal to increase the area of the active functionalized zone. For example, the size of biological cells is very often much larger than the integrated elementary devices.

\section{New Challenges for Electronic Systems}

Two main types of challenges are emerging on the horizon. The first concerns the technical, technological and physical aspects, the second is related to human resources. Future engineers and technicians will have to face these technical and technological challenges.

\subsection{Technical and Physical Challenges}

Since 2005, the development in the world of connected objects and IoT has been incredible. Figure 2 illustrates this evolution. The growth is exponential and their number is more or less doubled every 4 years. The number of connected objects follows the same exponential law [16].

Let us notice that the number of sensors follows also an exponential growth. Indeed, their number are expected to double every two years, also [12].

As a consequence, the industry is becoming more and more competitive in the market-place. Thus, the design and manufacture of new connected objects must be carried out in an increasingly short time. Thanks to intellectual property (IP) modules, the microelectronics companies may design faster and faster new products involving assembling of several electronic functions similarly to Lego game. However, there are not optimized in terms of circuit size, clock frequency, and the number of elementary devices by function. In parallel, even if the technologists have realized a lot of improvement, due to the reduction of dimension close to atomic scale, the leakage currents are becoming relatively much higher and the losses in commutation too. As a result, the size of the electronic part of the connected objects and the associated electrical consumption are very far from the new needs in terms of consumption of natural resources and energy for both manufacturing and operation.

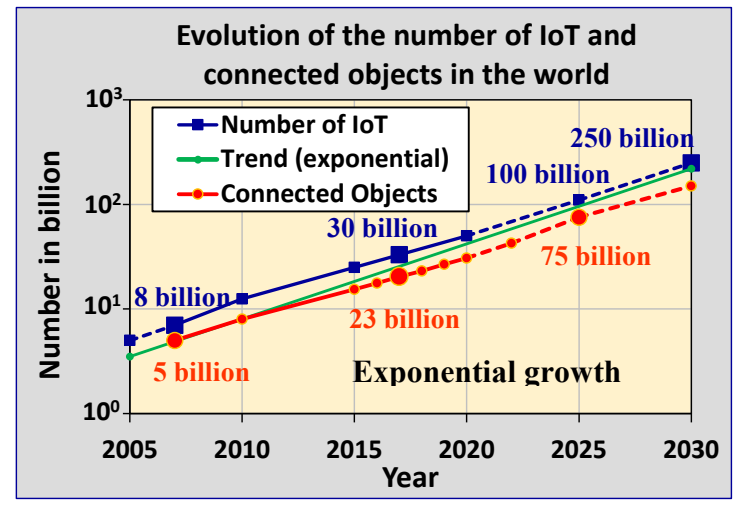

Figure 2. Evolution of the IoT and connected objects since 2005. In both cases, the variation is exponential. Their respective numbers double every four years. 
Thus, a very important new challenge is due to the growth in energy consumption worldwide due to the exponential growth in the number of connected objects and IoT. Figure 3 shows the evolution of overall energy consumption if the behavior of connected object manufacturers does not change in the near future. In 2018, the IoT's global consumption of electrical energy was equivalent to three times the global air traffic, or about $2900 \mathrm{TWh}$, which corresponds to a permanent electricity consumption of $325 \mathrm{GW}$. But its evolution is also exponential. The International Energy Agency forecasts a growth rate corresponding to a doubling of this consumption every four years [17].

By 2040, without modification of the behavior at the global level, IoT consumption could reach the global energy consumption of 2018, regardless of the field of use. The challenges concern all facets of the microelectronics field and more particularly all the circuits and systems that make up the connected objects. The innovation challenge must now succeed in reducing the energy consumption of basic electronic components, modifying circuit architectures to reduce the instantaneous consumption of billions of transistors, extending standby operations (or idle) to minimize the energy consumption of electronic systems, minimizing Internet use by promoting local communications (low power transmission-reception) and replacing massive silicon or semiconductor integrated circuits with thin film devices that are mostly much less energy consuming. Power devices and power electronics are also concerned in order to improve the yield of electrical conversions (AC-DC, high voltage-low voltage).

\subsection{Human Resources Challenges}

The technical and technological challenges are therefore very important and diversified. At the global level, there is a need for skills and competencies. In other words, we must train young graduates in all aspects of microelectronics, including thin-film and large-surface technologies. Training both on knowledge in general and on know-how is becoming a challenge. There is a need to train more

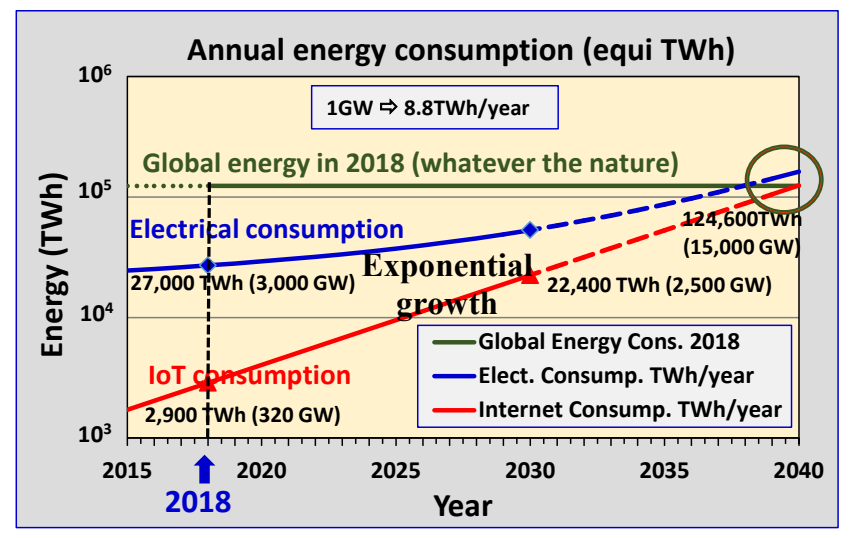

Figure 3. Evolution of the electrical energy consumption of the IoT since 2005. In both cases, the variation is exponential. The energy consumption doubles every four years. By 2040, without modification of the behavior at the global level, the energy consumption will reach the 2018 global energy consumption regardless of the field of use [17]. 
engineers and technicians at the production level and more doctors who can ensure research and development and innovation in all the areas mentioned above [18]. Let us notice that, even for the industrial production the fourth industrial revolution will need more and more skills for involving robots and connected manufacturing.

The problem is therefore to train students through practical training on dedicated platforms. This requires dedicated teaching platforms, accessible to students in initial training at all levels but also in lifelong learning, at a limited cost and in line with the needs of industry, teaching and research.

In France, a network has been in place since the early 1980s to create training centers dedicated primarily to education with modern platforms adapted to the constant evolution of the field. This network, entitled "CNFM" for National Coordination for Training in Microelectronics and Nanotechnologies [19] is composed of twelve interuniversity centers and two industrial partners who are in fact industrial unions [20].

These academic centers have technology and design platforms, including seven clean rooms that accommodate students from all universities and schools in a region, and a national computer-aided design service. This network is recognized by the Ministry of Higher Education. The industrial unions provide information and advices on the industrial and economic trend [21]. Strong links are thus permanently established that also encourage innovation.

Annually, this network receives 17,000 students on its eighty platforms spread over 12 centers. In order to maintain a quality of training adapted to economic needs, the network has been pursuing an innovation policy for more than ten years by issuing calls for proposals every year. Since 2012, an incentive program for innovative training as part of the French government's excellence initiative program has reinforced this strategy. The eight-year project, entitled FINMINA [22], has been driving the innovative strategy since 2012 and has enabled new practical activities to be implemented, particularly in the field of large-area electronics and thin-film transistors. Depending on the local research and industrial environment, the centers have specialties. The network can then propose a variety of subjects best suited to the skills of the teaching teams and the equipment of the technological platforms.

In order to correspond to evolution, students can work on the realization of elementary components such as CMOS like TFTs, but also on original structures that are sorted in thin film technologies, such as polycrystalline silicon thin film transistors [23], thin film airgap transistors for sensor development [24] [25] or transistors using two-dimensional material layers [26]. These structures are issue of research activities in the close environment of the training center. Several student projects propose to go as far as the system including the packaging steps corresponding to SiP development. For these activities, all aspects can be addressed: component and circuit design, clean room manufacturing, physical and electrical characterizations, and testing of circuit, components or even systems. 


\section{Practice and Know-How on Thin Film Technologies}

\subsection{Importance of Practice and Know-How}

Innovation, research and development require engineers, technicians or $\mathrm{PhDs}$ to have both skills and know-how. It is therefore necessary to adapt the training of these research, development and production staff in order to make them operational at the end of their studies and after their diplomas. As mentioned above, large-area electronics and associated components are becoming increasingly important in integrated systems and especially in system-in-package ( $\mathrm{SiP}$ ), which corresponds to the stacking of circuits possibly on the same substrate but in any case in the same package.

\subsection{Know-How on Thin Film Technologies in the Centers}

Figure 4 shows several examples of devices carried out by students during practical training that has been implemented through the FINMINA project over the past six years. The components, mainly thin-film transistors (TFTs) in these examples [23], have been designed, technologically processed and characterized on the different platforms of the French network.

Figure 4 shows successively, polycrystalline silicon TFT involving a silicon oxide gate insulator for CMOS circuits [27], a TFT with Hall magnetic contacts

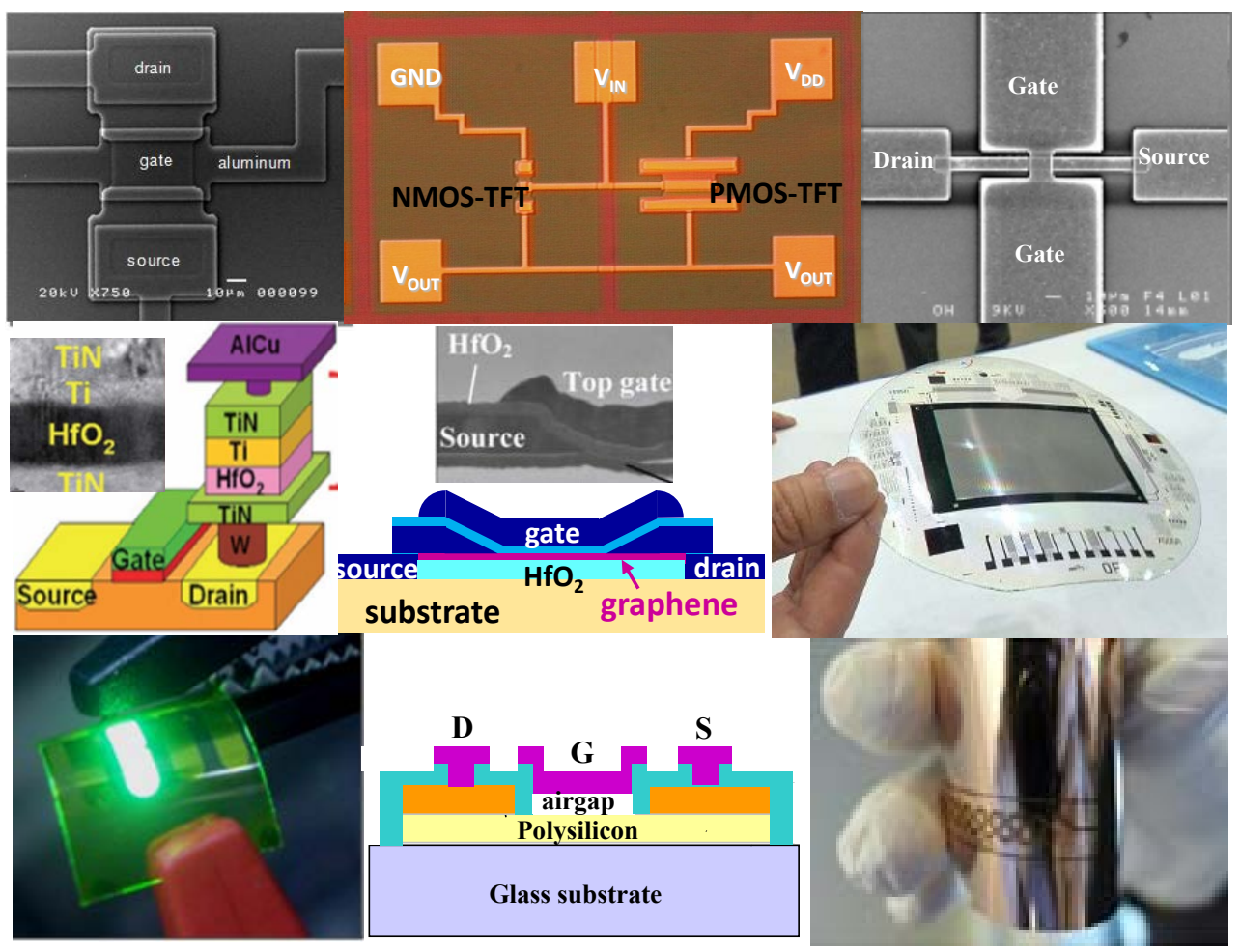

Figure 4. Examples of realizations of different TFT that can be involved in electronic circuits or in sensors made by students in the frame of initial education in the French national network centers. From top left to bottom right: polycrystalline silicon TFT, CMOS-like TFT circuit, airgap TFT, OxRam thin film memory, graphene-based TFT, flexible displays, organic light emitting diode, chemical sensor, and flexible thin film circuit. 
[28], a silicon nanowire TFT acting as a gas sensor [24], a TFT air gap for chemical and biological sensors [25], a graphene-based TFT [26], a flexible substrate TFT for wearable electronics.

\section{Conclusion}

Many challenges have recently arisen in the frame of the strong evolution of the society towards a digital world. The challenges are technological but also human. On the technological facet, the combination of many technologies is a way to spread the spectrum of application domains and to minimize the energy consumption. The main objective is now to train the students in an adapted environment that allows them acquiring the know-how. Only few examples were given of the effective work of the network in this paper [29]. All these projects were carried out by students as part of their initial training, at master's or engineer level. Several of them corresponding to a long manufacturing process are performed by a group of students as part of an internship. Each year, about 200 students acquire this know-how on thin film technologies in the frame of the French network [30]. We expect that they have been well prepared to develop innovative objects in the coming connected world by involving thin film technologies.

\section{Acknowledgements}

The author would like to thank all the members of the French GIP-CNFM network for their contribution to many innovative projects. This work is financially supported by the Ministry of Higher Education and by the IDEFI-FINMINA program (ANR-11-IDFI-0017). Many thanks to L. Chagoya-Garzon, Secretary of the GIP-CNFM for his wise advice in proofreading this paper.

\section{Conflicts of Interest}

The author declares no conflicts of interest regarding the publication of this paper.

\section{References}

[1] Burgess, M. (2018) What Is the Internet of Things? WIRED Magazine. https://www.wired.co.uk/article/internet-of-things-what-is-explained-iot.

[2] McKellop, M. (2018) The Future of the Semiconductor Industry Is the Internet of Things. The Burn-In, 28 November 2018.

[3] Chaouchi, H. (2010) The Internet of Things: Connecting Objects. ISTE Ltd, John Wiley \& Sons.

[4] Bonnaud, O. (2016) New Approach for Sensors and Connecting Objects Involving Microelectronic Multidisciplinarity for a Wide Spectrum of Applications. Int. J. Plasma Environmental Science \& Technology, 10, 115-120.

[5] Bonnaud, O. (2015) The Multidisciplinary Approach: A Common Trend for ULSI and Thin Film Technology. ECS Transaction, 67, 147-158.

https://doi.org/10.1149/06701.0147ecst 
[6] Santos, B., Charrua-Santos, F. and Lima, T.M. (2018) Industry 4.0: An Overview, Proceedings of the World Congress on Engineering 2018, Vol II.

[7] See for Example (2019). https://yearbook.enerdata.net/total-energy/world-energy-production.html

[8] Swaminathan, M. and Pettit, J.M. (2011) 3rd System Integration Workshop.

[9] Moore, G.E. (1965) Cramming More Components onto Integrated Circuits. Electronics Magazine, 38, 114-117.

[10] Simonite, T. (2016) Moore's Law Is Dead. Now What? MIT Technology Review, https://www.technologyreview.com/s/601441/moores-law-is-dead-now-what/

[11] Sharma, V. and Tiwari, R. (2016) A Review Paper on "IOT" \& It"s Smart Applications. International Journal of Science, Engineering and Technology Research (IJSETR), February 2016, 5(2).

[12] Schütze A., et al., (2018) Sensors 4.0-Smart Sensors and Measurement Technology Enable Industry 4.0. J. Sens. Sens. Syst., 7, 359-371. https://doi.org/10.5194/jsss-7-359-2018

[13] Bonnaud, O. (2020) Mandatory Matching between Microelectronics Industry and Higher Education in Engineering toward a Digital Society, Smart Education and e-Learning 2019. Part of Springer Nature Singapore Pte Ltd., Chap. 24, 255-266. https://doi.org/10.1007/978-981-13-8260-4_24

[14] Bonnaud, O., Bsiesy, A. and Pichon, L. (2018) Thin Film Transistor Technologies: Implementing Research Results in Higher Education to Prepare the Emerging Multidisciplinary Connected Object. Elec.Chem.Soc. Trans., 86, 3-10. https://doi.org/10.1149/08611.0003ecst

[15] Chaker, A., Gonon, P., Vallée, C. and Bsiesy, A. (2017) High Capacitance Density of $185 \mathrm{nF} / \mathrm{mm}^{2}$ Achieved in Three-Dimensional MIM Structures Using $\mathrm{TiO}_{2}$ as a Dielectric. Appl. Phys. Lett., 110, 243501. https://doi.org/10.1063/1.4986196

[16] After (2019). https://fr.statista.com/statistiques.

[17] Source (2019) International Energy Agency. https://www.iea.org

[18] Bonnaud, O. (2019) New Vision in Microelectronics Education: Smart e-Learning and Know-How, a Complementary Approach. In: Uskov, V., et al., Eds., Springer Nature 2019 KES-SEEL-18 2018, Springer International Publishing AG, SIST 99, 267-275. https://doi.org/10.1007/978-3-319-92363-5_25

[19] GIP-CNFM; Public Interest Group-National Coordination for Education in Microelectronics and Nanotechnologies. http://www.cnfm.fr

[20] ACSIEL Alliance Electronique (2019) Components and Systems Alliance for Electronics Industry in France. http://www.acsiel.fr/en-GB/index.aspx

[21] Matheron, G. (2014) Microelectronics Evolution, Keynote. European Microelectronics Summit, Paris (France).

[22] FINMINA (2019) Formation Innovante en Microélectronique et Nanotechnologies, (Innovative Training for Microelectronics and Nanotechnologies). IDEFI Project: ANR-11-IDFI-0017.

[23] Pichon, L., Raoult, F., Bonnaud, O., Sehil, H. and Briand, D. (1995) Conduction behavior of Low Temperature $\left(\leq 600^{\circ} \mathrm{C}\right)$ Polysilicon TFT with an In-Situ Drain Doping Level. Solid State Electronics, 38, 1515-1521. https://doi.org/10.1016/0038-1101(94)00277-m

[24] Mahfoz-Kotb, H., Salaün, A.C., Mohammed-Brahim, T. and Bonnaud, O. (2003) Airgap Polysilicon TFT for Sensor Application. IEEE ED Let., 24, 165-167. 
https://doi.org/10.1109/led.2003.809536

[25] Bendriaa, F., et al. (2006) Sensitivity of Suspended-Gate Polysilicon TFTs to Charge Variation and Application to DNA Recognition. ECS Transactions, 3, 341-347. https://doi.org/10.1149/1.2356372

[26] Happy, H. (2014) Invited Paper, Electronics Exhibition ENOVA. Lyon (France).

[27] Crand, S., Gautier, G. and Bonnaud, O. (2003) Dynamic Electrical Characterization of CMOS-Like Thin Film Transistor Circuits. Proc. of IEEE Int. Conf MSE2003, 14-15. https://doi.org/10.1109/mse.2003.1205233

[28] Le Bihan, F., et al. (2001) Realization of Polycrystalline Silicon Magnetic Sensors. Sensor \& Actuators, 88, 133-138. https://doi.org/10.1016/s0924-4247(00)00506-9

[29] Bonnaud, O. and Fesquet, L. (2016) Practice in Microelectronics Education as a Mandatory Complement to the Future Numeric-Based Pedagogy: A Strategy of the French National Network. Proc. EWME2016, Southampton (UK), May 2016, 1-8. https://doi.org/10.1109/ewme.2016.7496460

[30] Bonnaud, O. and Fesquet, L. (2016) Innovation in Higher Education: Specificity of the Microelectronics Field. Proc. of IEEE SBMicro 2016, Belo Horizonte (MG-Brazil), 1-4. https://doi.org/10.1109/sbmicro.2016.7731342 